\title{
ZOOMING IN AND OUT: STUDYING PRACTICES BY SWITCHING THEORETICAL LENSES AND TRAILING CONNECTIONS ${ }^{1}$
}

\begin{abstract}
This paper contributes to re-specifying a number of the phenomena of interest to organisational studies in terms of patterns of socio-material practices and their effects. It does so by outlining a vocabulary and strategy that make up a framework for theorising work and organisational practices. The vocabulary is based on number of sensitising concepts that connote practice as an open-ended, heterogeneous accomplishment which takes place within a specific horizon of sense and a set of concerns which the practice itself brings to bear. The strategy is based on the metaphorical movement of "zooming in" and "zooming out of" practice. The zooming in and out are obtained through switching theoretical lenses and repositioning in the field, so that certain aspects of the practice are fore-grounded while others are bracketed.

Building on the results of an extended study of telemedicine, the paper discusses in detail the different elements of the framework and how it enhances our capacity to re-present practice. The paper concludes with some considerations on how the proposed approach can assist us in advancing the research agenda of organizational and work studies.
\end{abstract}

Keywords:

Practice theory

Organisational theory

Processualism

Relationalism

Telemedicine

Study of work

\footnotetext{
${ }^{1}$ To appear in Organization Studies, 30(12) 2009. I am in debt to Maxine Robertson and Frank Blackler who read and generously commented on previous versions of this manuscript and the work on which it is based. Many thanks also to my colleagues at IKON and the editors of the special issue for their suggestions and guidance. As customary, all the responsibility for ignoring some of their valuable advice rests firmly with the author. Finally I would like to acknowledge the precious editorial support of Adam James.
} 


\section{INTRODUCTION}

One of the most visible and intriguing recent developments in organisation studies is the increased interest in the detailed understanding of how real-time practices are carried out in the workplace and the relation between workplace activities and the organizing process. This interest, which parallels a generalised renewed interest for practice in social science (Ortner, 1984; Schatzki et at, 2001), feeds on two concerns, which are distinctive to this particular disciplinary field.

First, the so-called 're-turn to practice' in organisation and management studies (SamraFredericks, Miettinen and Yanow, 2005) stems in part from a reaction to a previous marginalisation of the study of 'work itself' by significant sectors of this research community. The renewed interest in practice can thus be interpreted as an attempt to reground the theorising in "what is actually done in the doing of work and how those doing it make sense of their practice" (Orr, 1998, p. 439) and closing "the chasm between practicedriven theorising of what people do in their workplace and academic theory-driven theorising about it" (Yanow, 2006, p. 1745).

Second, the idea of practice is particularly appealing as it promises to re-specify a number of the phenomena that constitute the object of work of organisation studies - from hierarchy to inequalities, from knowledge to innovation and change - in terms of a complex array and nexus of socio-material activities and their effects. The essential idea is that practice constitutes the site of organisation and that organisational phenomena transpire through, and are effects of, a texture of interconnected practices (Schatzki, 2002, 2005; Reckwitz, 2002; Czarniawska, 2004; 2007). The basic domain of study of organisational studies becomes thus "the pattern of social and material practices of organising ordered across space and time" (Giddens, 1984, p. 2).

Adopting real-time practice as the starting point of social and organisational inquiry, however, poses a clear difficulty. As authors such as Heidegger (1947) and Wittgenstein (1953) made clear, practice constitutes the unspoken and scarcely notable background of everyday life. Practices therefore always need to be drawn to the fore, made visible and turned into an epistemic object in order to enter discourse. In what follows, I will present some preliminary indications on how this can be achieved and how we can go about 
theorising practice. The main purpose of the paper is, therefore, to outline a provisional framework for studying, analysing, and re-presenting practice.

Using examples from an extensive study of telemedicine, I will in particular suggest that for understanding and re-presenting practice we need first to "zoom in" by selectively introducing a variety of conceptual tools and perspectives which can help us appreciate different aspects of it. For theoretical and methodological reasons which I discuss in the paper, the starting point for this zooming in on practice should be the detailed study of its discursive and material accomplishment. This, I will argue, is however only part of the job. A coherent practice approach needs also to address how translocal phenomena come into being and persist in time as effects of the mutual relationships between the local real-time accomplishments of practices, as well as how they make a difference in the local process of organising. For theorising practice, we need an appropriate methodological approach that makes us see the connection between the here-and-now of the situated practicing and theelsewhere-and-then of other practices. I will describe this second movement as "zooming out" of practice. Theorising practice thus requires a double movement of zooming in on, and zooming out of, practice obtained by switching theoretical lenses and trailing the connections between practices. The framework and methodological dispositions, which I present here in preliminary form, will enable us to understand both the conditions of the local accomplishment of practice and the ways in which practices are associated into broad textures to form the landscape of our daily (organisational) life.

The article is organised as follows. After briefly positioning my effort in the extant literature, I introduce a number of sensitising concepts and methods that can assist us in the zooming in and the study of the local accomplishment of practice. This is followed by a discussion of the logic and practicalities of studying the zooming out by following connections in action. The concluding remarks reiterate some of the central tenets of the framework and discuss some of the ways in which the proposed approach can assist us in pursuing a coherent nonfunctionalist research agenda within organisation and work studies.

\section{POSITIONING THE EFFORT: THEORY AND METHODOLOGY}

\section{Theoretical tributaries}


On reading the rest of the paper, the reader will realise that several theoretical traditions are echoed in the idea of the zooming in and out. Four approaches in particular constitute the main tributaries of my framework and need to be briefly mentioned here.

First, I draw on the Wittgenstenian and Heideggerian view of social affairs and their emphasis on the centrality of social practices and practical understanding as the foundational texture of everyday life (Schatzki, 1996; 2002; Shotter, 1993; Chia and Holt, 2006). These two traditions take practices as primitive and argue that mutual understandings, systems of beliefs and rules build upon on them and rely on their existence. It follows that the meaningful, purposive and consistent nature of human conduct descends from participating in social practices and not from the deployment of rules, goals and beliefs. The Wittgenstenian and Heideggerian traditions therefore allow us to decentre such phenomena as mind, meaning and intentionality, so locating the roots of social co-existence in the practices that all people qua humans are necessarily involved (Schatzki, 1996; 2002; Chia and Holt, 2006).

Second, I build on the ethnomethodological intuition that organised activity needs to be studied in terms of the methodical practices used by competent members to assemble concerted scenes of action (Garfinkel, 1967; Boden, 1994; Luff et al., 2000; SamraFredericks, 2003; 2005; Llewellyn and Hindmarsh, 2009). From the rich and multifaceted ethnomethodological tradition, I derive, among others, the principle that treating practice in descriptive terms is often not enough and that a coherent analytical stance is necessary for the goal of outlining a coherent practice-based ontology (Llewellyn, 2008). Once we have accepted that the world is the result of an incessant process of social construction, we have only begun our task. Claiming that social structures, inequalities, power and meaning are "constructed" requires that we provide convincing accounts of what this means in practice, e.g., by specifying the methods and devices used to obtain such effects (Lynch, 1993). Third, I draw extensively on Cultural and Historical Activity Theory (Engeström, 1987; 2000; Blackler, 1993; Miettinen, 2005). A central tenet of this approach is the idea that all social and material practices emerge around an object or prospective outcome that "motivates and directs activities, around which activities are coordinated, and in which activities are crystallized (...) when the activities are complete" (Kaptelinin and Nardi 2006: 66). While many aspects of practice (from the division of labour to the rules and tools to be used and the identity each member will assume) emerge around a specific object of work, such an object is also the result of the interests of the community that gathers around it plus other interests mediated in the activity through a variety of other intermediaries (Miettinen and Virkkunen 2005). Accordingly, all practices embody different interests and are hence internally 
fragmented, subject to multiple interpretations, and open to contradictions and tensions. This, in turn, makes all practices necessarily tentative and ever-changing.

Finally, I build on Actor Network Theory (ANT: Latour, 2005) and other semiotic-orientated social ontologies (Czarniawska, 2004; Czarniawska and Hernes, 2005, Lindberg and Czarniawska, 2006; Czarniawska, 2007). These approaches draw attention to the constitutive power of associations. They argue that social agency (both individual and collective) is constituted through assembling, aligning and stabilising patterns of relationships so that any form of social order is in fact the outcome of observable instances of ordering. From this follows that the main task of social science is tracing the associations between human and non-human elements and studying the effects that the resulting arrangements make in the world. By emphasising that any form of social order, no matter how seemingly 'macro' and durable, is the result of the active connection between local instances of ordering, semioticoriented social ontologies offer both a language and a method for studying organisational and institutional phenomena without having to revert to the idea of pre-existing levels of reality. Although they are not strictly speaking theories of practice, ANT and the sociology of translation offer a powerful theory/method package for outlining a thorough practice-based ontology of organisational phenomena.

In the rest of paper I draw on these traditions (and a few others that I do not discuss here for reasons of space $^{2}$ ) to outline a framework for theorising work and organisational practices. Although they belong to different traditions, these theories can be in fact mobilised together in that they all subscribe to some key common tenets. For example, all these theories are joined in the belief that:

- Practices constitute the horizon within which all discursive and material actions are made possible and acquire meaning; that practices are inherently contingent, materially mediated, and that practice cannot be understood without reference to a specific place, time, and concrete historical context (Engeström, 2000; Latour, 2005; Schatzki, 2002; 2005).

- While practices depend on reflexive human carriers to be accomplished and perpetuated, human agential capability always results from taking part in one or more socio-material practices (Reckwitz, 2002).

\footnotetext{
${ }^{2}$ Readers will also recognise, for example, the influence of the work of Bourdieu (1980), Giddens (1984) and the study of discourse as practice (Iedema, 2007).
} 
- Practices are mutually connected and constitute a nexus, texture, field, or network (Giddens, 1984; Schatzki, 2002; 2005; Latour, 2005 Czarniawska, 2007). Social coexistence is in this sense rooted in the field of practice, both established by it and establishing it. At the same time, practices and their association perform different and unequal social and material positions, so that to study practice is also the study of power in the making (Ortner, 1984).

The reason for tapping into multiple traditions is that each of them offers both advantages and limitations for the study of practice. For example, both ethnomethodology and the Wittgenstenian tradition have very little to say about the central role of objects and about the evolving nature of practice - all concerns that are central in the Cultural and Historical Activity Theory tradition. In a similar way, while ethnomethodology provides a rich a sophisticated tool-kit for investigating practice "as it happens", it only takes into consideration phenomena such as social structure, institutions, and power to the extent that they visibly manifest themselves in the actual social practices of members (Garfinkel 1967; Garfinkel and Sacks, 1970; Hilbert, 1990; Samra-Fredericks, 2004; Llewellyn, 2008). In this sense, the approach is scarcely adequate to evoke a positive relational organisational ontology whereby translocal phenomena, seen as the result of the interlacing of local instances of order production, acquire the capacity to retroact at the local level. This in turn requires an integration of the ethnomethodological sensitivity with the intuition of semiotic-oriented social ontologies such as Actor Network Theory and the sociology of translation (Czarniawska and Hernes, 2005), two approaches particularly useful for conceptualising and studying 'macro' phenomena as the result of the association between local instances of ordering.

In short, the rationale for my intended eclecticism is programmatic: to the extent that practice is a multifaceted and multi-dimensional phenomenon, it can only be approached through a toolkit-logic and a collage, heteroglossia, or even carnivalesque, approach (Bakhtin, 1981; Belova et al, 2008).

\section{The case study}

This multiplicity of influences and my underlying relational ontology are mirrored in the methodology used to generate the telemedicine example used in the paper to illustrate my views. The data derive from a three-year ethnographic and interpretive investigation 
conducted by the author in northern Italy ${ }^{3}$. During the project - and at different points in time - I observed for extended periods (days or a week) the practice in the context of the daily ward's routine and followed nurses in their daily activities. I attended meetings, promotional workshops and training sessions; conducted and tape-recorded about 50 ethnographical, semi-structured, and other styles of, interviews, with doctors, nurses, managers and health officials (some of these interviews were conducted with the help of another researcher); collected and analysed documents, tools and pictures; and travelled to other sites where people were trying to apply the same, or other forms of, telemedicine. As I discuss in depth elsewhere (Nicolini, 2009b), the study alternated between a focus on the accomplishment of telemedicine in specific places and an attempt at making sense of the associations between different local accomplishments of the practice and other more distant activities. Accordingly, the empirical material presented in the rest of the paper was obtained by combining elements of focused ethnography (Knoblauch, 2005) and micro-ethnography (Streeck and Mehus, 2004) with the application of ANT's main precept to "follow the actors themselves" (the "actor" being, in this case, the practice of telemonitoring: see Latour, 2005 for a discussion). While the former responded to the need of understanding in depth the local accomplishment of the practice itself, the latter derived from the empirical finding that telemedicine, like many other aspects of modern organising, happened 'in many places at once' (Czarniawska, 2007, p. 16). Telemedicine could thus only be studied through some form of 'mobile ethnology' based on materially investigating the trails of connections between practices and their products. In this way, the study slowly extended from the original site to other centres and locations which had become relevant for the understanding of the new ways of caring for patients. The study thus ended up being both multi-method and multisited (Marcus 1995; Hannerz; 2003), an attempt both to understand the emergence of telemedicine and to explore which tools are appropriate for doing fieldwork when the 'field' is interpreted as a 'field of practice' (Czarniawska, 2007, p.7).

\section{The contribution}

It is against the above backdrop of multiple theories and methods that I would like to claim the incremental difference of my approach vis-à-vis that of others. My fundamental assumption, implicit in the idea of zooming in and out, is that studying practice requires

\footnotetext{
${ }^{3}$ Other aspects of this case are dicussed in Nicolini, 2006, 2007. Financial support for this research was provided in part by the Provincia Autonoma of Trento (Italy), Progetto Scientifico No. 6-2001.
} 
choosing different angles for observation and interpretation frameworks without necessarily giving prominence to any one of these vistas. My effort is, therefore, one of sequential selective re-positioning ${ }^{4}$.

It follows that both the strength and originality of my contribution stems largely from the attempt to combine existing insights, rather than introducing a totally new approach. Unlike other authors ${ }^{5}$, I am in search of an eclectic set of sensitising concepts that allow different features of practices and their associations to come to the fore while others are suspended. The zooming movement through which I propose to re-present practice is achieved, in fact, by switching theoretical lenses and trailing connections according to a set of specific assumptions. The act of zooming in and out should be interpreted as foregrounding and backgrounding boundaries in the programmatic attempt to complexify practice against all types of reductionism, including what Levinson (2005) calls "interactional reductionism" (the tendency of reducing all social phenomena to local interaction). The guiding image that I would like to outline within the limit of a single paper is one of organising as the effect of a seamless web of local practices (Latour, 2005) or an action net which extends in space and time (Czarniawska, 2004; Lindberg and Czarniawska, 2006). From this perspective, both the instances of local production of organised activity, their associations and the effects produced by their being associated in one way rather than another constitute relevant objects of inquiry. In the next sections I will outline my proposed theoretical and methodological framework starting by illustrating the zooming in and proceeding to describe the zooming out movement. I will use my empirical examples both to clarify my theoretical claims and to illustrate the range of methods that might be appropriate to a zooming in/out approach, some of which I productively used in my empirical research.

\footnotetext{
${ }^{4}$ I owe this expression and several others to the perceptive comments of the anonymous reviewers of previous versions of this paper. I would like to thank them for their precious contributions.

${ }^{5}$ Although my work here bears similarities to the idea of appreciating activity by switching theoretical lenses (Samra-Frederick, 2003; 2005) and scaling-up and bearing down in the analysis of discourse (Hardy, 2004), my project is different in at least two important ways. Firstly, my attempt is to usher in a variety of theoretical sensitivities and theories of practice that are different from those suggested in the previous literature. Secondly, unlike some of these authors, my project is not to provide an ethnographic contextualisation of the ethnomethodological study of practice but to provide an eclectic framework that can integrate the prescription of ethnomethodology.
} 


\section{IN THE BEGINNING WAS THE DEED}

One of the main characteristics of the approach sketched above is that it requires an endorsement of a particular qualitative and interpretive research approach sensitive to the material, factual, and temporal nature of practices. Gubrium (1988) calls "articulative" this type of approach that is endorsed by many of the authors mentioned in the previous section. Unlike other approaches, an articulative sensitivity directs attention towards the modes of accomplishment of real-time practices and, in fact, practicing is formed anew each time. In order to exemplify the type of epistemic objects generated by an articulative style of inquiry, in the next section I shall contrast a manual-like, "canonical" (Orr 1996) account of making scheduled telemonitoring telephone calls with fragments of a non-canonical description ${ }^{6}$. The canonical description is derived from official documents produced by the practitioners to describe the new practice for scientific and administrative purposes. This will be followed by an articulative description derived from my large study mentioned above. I will start by providing a very brief description of what is involved in "doing being a nurse" and making a telemonitoring call. I will then briefly illustrate what it means to follow a schedule and to work collaboratively for addressing some of the technological limitations of this way of doing thing -namely how to eliminate some of the interferences produced during the transmission of data. The few fragments, which derive from my filednotes, presented are of course not exhaustive, and they are mainly used to exemplify my points.

The practice of telemonitoring is a relatively novel method of caring for patients with serious chronic heart failure (CHF) from a distance. Using telephone calls - a combination of scheduled calls (telemonitoring) and advisory calls (tele-assistance) staffs gather clinical data, monitor patients, and take necessary corrective action to maintain patients in a stable condition. These actions might range from reassuring patients, to modifying their existing therapy, to sending an ambulance to the patient's address. In this way patients can remain at home, and visits to accident and emergency departments can be reduced, so freeing up hospital beds.

\section{A canonical description of scheduled contacts}

\footnotetext{
${ }^{6}$ I have drawn the idea of comparing a canonical/distal and a practice-based account of work from Orr (1996) and Crabtree (2001).
} 
[From an official document]

- All patients are given a portable monitor which transmits a single-lead electrocardiography trace by phone; they are also told the numbers to call.

- Upon receiving the call, the nurse will analyse the single-lead ECG, comparing it with the benchmark basal trace on record to demonstrate or to exclude the presence of arrhythmias or changes in heart rate.

- Telephone contact will be fortnightly for patients with mild/medium symptoms and weekly for patients with a serious heart condition. The timing of the contacts might change according to a patient's condition and based on the clinical data collected each time.

- During the scheduled telephone call the nurse shall assess the presence of clinical decompensation and will check the drug therapy, recording any variation of dosage. The nurse will also attend to the health training of the patient and deal with any requests.

- During each call the following information will be gathered: degree of clinical stability (if the patient is unstable, symptoms and signs of decompensation will be recorded); therapy; access to hospitals (date, cause, duration); access to accident and emergency units (date and cause); specialist examination; encounters with own family doctor; blood tests; death (data and cause)

\section{A practice-based view of telemedicine practice}

\section{Doing "being a nurse"}

...Every nurse at G. knows that you report to work promptly at 8am, go to the cardiology ward and put on your uniform... You move on to the telemedicine room...The first thing to do is to reply to urgent requests. If patients have called during the night, you must call them back immediately. Only when you have no other commitments can you start to make your phone calls. You make the quickest calls first. These are calls where you check on the patient's health, collect data not sent that day or previous days, or ask when the next check-up will be. You will remember that one of the fundamental aspects of your work is to "show that you are there". So it is better to make one call more than one less. In addition, if the patient is not at home, you note this in your agenda and when you do manage to contact them 
you inform them that you called previously. You must always use the agenda, particularly because it can back you up if someone complains that you did not call...

\section{Making a call}

Imagine the following. The nurse is sitting at her desk. She has placed her agenda and two large ring binders with clear plastic envelopes in front of her. She opens one of the two binders and retrieves that belonging to the patient she wants to call. She turns and takes two fax sheets from a pile on her desk: an ECG trace received earlier and the results of a patient's laboratory tests. She takes a ruler from her pocket, measures the pulsating curve of the electrocardiogram and shakes her head. She then reaches for an A3-sized sheet that she places on the table. This "therapy sheet" contains a number of columns to record the date of the call, heart function, pressure, weight and dosage of each drug taken by the patient. The nurse briefly looks at the arrangement of documents in front of her and then calls the patient:

Insert table 1 about here

The nurse needs to ensure that the data are not only truthful, but also useful and meaningful. The usefulness of the data depends on how these data have been obtained - something that the nurse is keen to find out. Because water retention is a typical symptom of CHF, knowing when the body weight has been measured makes a significant difference. The overall meaning of the data (whether or not the increase of weight signals an incipient failure) is obtained by establishing connections between the readings and the previous data and by framing the information obtained within the background provided by information on particular conducts or events. The reason why the patient hurries, albeit reluctantly, to inform the nurse of her excessive eating, is that she knows her blood readings are out of the accepted range - they are much higher than the previous reading. If the reading is due to "unexplained" causes, it could signal a sudden deterioration of her health. This is not the case in this instance.

\section{Following schedules}

The two nurses in the room are ready to make their planned morning telephone calls. 
It is a hot summer. The nurse looks at her diary and picks up the folder with the patient's documents. But then she hesitates. She puts the folder down and speaks aloud, as if talking to herself: "no point calling Mr. F. then... he always go for a walk with his wife when it is still cool....and he probably went to the family doctor as well... so he won't be home for sure. I will call this afternoon after his rests..." She picks up her agenda and writes a line in the afternoon section.

\section{Collaboratively eliminating 'artefacts'}

The nurse picks up one of the ECG traces she received earlier. She looks at it and shakes her head. She turns towards me and says “...see, this ECG is full of artefacts...” Pointing to some of the lines on the paper, she continues: "see some of these lines? They are disturbances from the recorder...this means that it was not positioned well on the chest...all the four stems need to touch the stern...this trace is unusable... too messy...I'll have to work again on this with Mrs. R."

The next day the nurse calls Mrs. R. "Hello, how are you this morning..." The nurse explains that she did receive the ECG trace, but it was "full of artefacts" (the patient seems to know what she is talking about!). "Do you mind if we go over the procedure one more time?" The nurse again explains how to position the recorder on the chest. The patient explains what she is doing ("here, I am putting it where you told me last time when I visited you"). The machine emits its rhythmical sound. At a certain point the sounds changes. The nurse exclaims: "Can you hear that? You have moved the recorder too much and now I am...we are getting artefacts!” The patient acknowledges this and moves the recorder. The original beeping sound returns. The nurse invites the patient to shift the recorder again so that she can "learn how to hear the artefacts" herself. Artefacts are thus repeatedly produced and recognised. The nurse reassures the patient "Mrs. R., there is nothing to be worried about, this new machine I gave you is handier to use but a bit tricky...the other one [which had an adhesive sensor which was applied to the skin] was easier to use...I know, but if you have any problem just call me...I promise it will not take long to learn..."

\section{Re-presenting practice through zooming in on the sayings and doings}

The contrast between the two textual productions of the preceding sections bears witness to the fact that different sets of assumptions and textual practices generate dissimilar epistemic objects. Such differences do not depend on the close proximity to the practicing itself: the 
first account was, after all, produced by the practitioners. The difference and unequal capacity to re-present practice in some of its depth and complexity lies, instead, in the re-presentation strategy employed.

The articulative description provided above is particularly helpful to illustrate my first tenet that the study and theorisation of practice must start with zooming in on the real-time practicing as an organised set of doings and sayings carried out using a variety of tools and mediatory resources. What distinguishes a practice-based approach is that what in traditional accounts appears as a given is seen and described here as a skilled accomplishment. The tools and cumulative practical experience of organisational ethnography (Ybema et al, 2009), and especially micro-ethnography (Streeck and Mehus, 2004) and organisational ethnomethodology (Luff et al, 2000; Llewellyn, 2008; Rawls, 2008; Llewellyn and Hindmarsh, 2009) are precious resources here. Micro-ethnography and ethnomethodology urge us, in fact, to understand and describe practice as a publicly available accomplishment based on the situated assembling of a number of discursive and non-discursive practices. They insist that enumerating the resources that enter into this accomplishment, e.g., rules, formal descriptions, categories, narratives, technologies and other artefacts - as, for example, in the first extract - is not sufficient. The description of practice requires that we capture the actual work that goes into any practice. In this sense, the style of inquiry and writing exemplified in the second series of extracts make us appreciate the mastery that goes into the accomplishment of a telephone call (from the use of a particular vocabulary and choice of lexicon, to the skilful management of the tempo and timing of practicing) in a way that escapes the canonical rendition. It also highlights that such features need to be obtained through specific doings and sayings.

The articulative approach moreover foregrounds that mastery transpires not only through the saying and what is said, but also in the doing and what is done. This aspect is here evoked by the graphic device in table 1 . The right side of the table re-presents, in fact, aspects of the body choreography through which telemonitoring is achieved. The paired descriptions of what the nurse does with her talking and with her body suggest, in particular, that the discursive and the non-discursive aspects blend seamlessly (Reckwitz, 2002). Practices have both a material and a discursive dimension: the saying is a way of doing as much as the doing is in what is said or not said. When it comes to practice, distinguishing material and discursive aspects has only an analytical, not a substantial value, and one should not give ontological priority to one over the other (Iedema, 2007). 
To the extent that practice is accomplished through organised sayings and doings, one could try to zoom in on the material at hand even further by bearing down on discursive practices (Hardy, 2004) using, e.g., the procedures and codification symbolism of conversation analysis. This would allow us to produce an additional, more fine-grained set of hypotheses on how telemonitoring is accomplished through discourse (see table 2. A list of symbols is provided in the appendix).

Insert table 2 about here

Using this type of notation and analytical strategy, we could appreciate, for example, that the overall exchange is structured through a turn-type pre-allocation typical of highly institutionalised medical encounters (ten Have, 1999). While the nurse maintains a caring, informal, and slightly playful tone (the beginning of the exchange is heard by the patient as playful teasing), the formality and institutionality of the interaction is silently performed by the recognisable patterns of talking in turns and the overall structural organisation of the exchange (Heritage, 2004). The nurse always talks first. She sets the scene for the next turn, and orients the conversation towards the specific (institutional) topics and features while excluding other possibilities such as small talk ("we are not here to chat", is what I often heard nurses say). When elaborated yet further ${ }^{7}$, such a type of analysis could help shed light on a variety of organisational effects, from institutionality to identity-making (Llewellyn, 2008).

It can be argued, however, that in spite of its increasing popularity, zooming in on practice in this way constitutes only one of the ways to produce a thicker re-presentation of practice. Increasing the level of granularity of the description and analysis using an ethnomethodologically-inspired conversation analysis approach is, in fact, just one option among many others. Moreover, the increase of granularity comes at a cost. The use of coding

\footnotetext{
${ }^{7}$ For instance, we could zoom in on the practical ways in which the nurse drills down the importance of following the dietary regime. Lines 2-7 of Table 2, for example, help detect the typical structural features of an instructional encounter (Mehan, 1985). In these situations, the person in the teaching position withholds the response during his or her turn, so eliciting an extended dialogue by the 'learner'. This continues until the symmetry between initiation and reply is re-established (this happens in line 8). In our case, the patient pauses three times (line 2) to allow for the nurse to regain control of the exchange. However, the nurse chooses to wait until the patient identifies the problem as the triglycerides, something the patient is aware of because she has seen the results of the test before faxing them to the nurse.
} 
procedures, such as those exemplified in table 2, while revealing increasing levels of detail, moves us away from the account of observable reportable practices. Instead, it leads us into a realm where, for example, corrigibility by members becomes almost impossible because of the high technicality involved, so that we are left with an account that is only "logically empirical” (Lynch, 1993).

Rather than zooming in by magnifying the data in the attempt to make visible aspects that are not viewable from a distance, we can opt for a different strategy. For example, we can zoom in on other and equally-important aspects, such as the objects used in the practice and their performative role. Or we can switch theoretical lenses and focus on the fact that accomplishing a practice is never a detached process, and that practicing is organised by a set of observable and reportable practical concerns. In both cases the zooming in is not obtained by putting the practice under the microscope. Rather, the zooming in consists of using theory to bring to the fore certain aspects while pushing others into the background. In the next sections I will examine in more detail some of these alternative ways of zooming in.

\section{Re-presenting practice through foregrounding the active role of tools and materials}

A different strategy for zooming in on and re-presenting practice, in line with several of the theories discussed in section 2, is to focus on its heterogeneous nature (Engeström, 1987; 2000; Latour, 2005). Representing practices without thematising the landscape of tools, artefacts and resources, which enter their accomplishments, and asking what they do and how they make a difference would produce, in fact, an impoverished and lacking account. A good example in this sense is provided by the therapy sheet used by the tele-nurses. This artefact was critical for the accomplishment of telemonitoring because it enabled the comparative reading of the data recorded from a number of telephone calls, allowing the patient's condition to be followed and the chronology of the patient's history to be reconstructed. As one of the nurses declared: "the therapy sheet was used as a guide for a long time ...more than that it guided our calls...especially when we were still inexperienced". The accomplishment of telemonitoring was thus attained thanks both to the mastery of the skilled human actors (as suggested by the ethnomethodological reading provided above) and the active contribution of a variety of tools and artefacts such as the therapy sheet. They both participated in and shaped the doing, although not necessarily in the same way. As I shall discuss more in depth later, these artefacts also mediated the result of activity carried out in different locations and time on the scene of action, thus both enabling and constraining the 
practice. Accordingly, zooming in on the non-human, as well as the human actants, is a critical step for understanding and re-presenting practice.

\section{Re-presenting practice through zooming in on its oriented and concerned nature}

Zooming in on telemonitoring activity can also be pursued along further theoretical axes that do not adhere to the assumptions of micro-ethnography and ethnomethodology. For instance, we could put to work the intuition of the Heideggerian tradition that to practice means always to care, or to take care of, something: practices are always oriented and they are performed in view of the accomplishment of the meaning and direction that they carry (Macintyre, 1981). For those who are involved in it, the accomplishment of a practice such as telemedicine is experienced as being governed by a drive that is based on both the sense of what to do and what ought to be done. Zooming in would require, in this case, to bring forward and to articulate the lived directionality and telos of the practice and to appreciate the fact that such an orientation is perceived in both cognitive and moral terms, so that "the force that governs [practices] is based on some conception of the good...the moral element is crucial..." (Thevenot, 2001, p. 59).

A good example of this paradigmatic feature of practice is the telephone call reported in table 1. The organized nature of the interaction derives in part from the fact that both the nurse and patient are tuned into the horizon of sense and the set of practical concerns sustained by the practice they contribute to sustain. This is what allowed the nurse to understand, without hesitation, the meaning of "90 over 130" (line 11 of table 1 and table 2). The example also nicely illustrates the moral character of the inner force that governs practices. In table 1 above the patient anticipates the nurse admitting an infraction when she says "you know...my triglycerides". By being socialized into the practice during her stay in hospital, she has become part of a pattern of ordering called telemonitoring which implies being absorbed into the moral sense of what is the inherent value of telemonitoring - which includes collaborating in the maintenance of certain parameters. Finally, the call foregrounds that the sense of what is going on, what is wrong, and what is the appropriate action to take next is experienced not in terms of abstract principles, goals or rules but more simply through a set of practical concerns.

Zooming in would imply, in this context, bringing forward such practical concerns which reflect the sense and direction of the practice and which govern - albeit non-causally - the production of sayings and doings. I say 'non-causally' to emphasise that I am not suggesting 
that the orientation of a practice and the practical concerns causally determines the conduct of its member as in the traditional functionalist approach. Practical concerns are, on the contrary, a way to describe the cogent way in which members experience the ordering capacity of practices - which implies that members still need to actively produce orderly patterns of sayings and doings using their learned competencies (i.e., they are not "practice dopes"). In this sense, my suggestion here comes close to one of the basic recommendations of CHAT, which suggests that to understand any form of social activity we need to foreground the object of work around which it unfolds. The object of work, in fact, “...give(s) actions their ultimate continuity, coherence and meaning” (Engeström, 2000, p. 964). What the notion of practical concerns adds to this idea is the need to describe how the object of work is experienced by members in the practicing ${ }^{8}$.

To articulate this aspect of practice we could make the most of the fact that practical concerns (and the object of work) are never held tacitly; on the contrary they are customarily addressed verbally and discussed in the course of the practicing, either through a vocabulary of motives and goals, or through a vocabulary of accounts, explanations, justifications, and prescriptions. By using the appropriate methods, we can thus capture and re-present them in a reportable and corrigible way.

For example, the extract in the section "Doing "being a nurse"" was obtained by using a form of qualitative interview called "the interview to the double" (Gherardi, 1995, Nicolini, 2009a). This is a technique that requires interviewees to imagine they have a double who will have to show up at their job the next day. The informant is then asked to provide the necessary detailed instructions that will insure that the plot is not unveiled and the double is not unmasked. Through this device, we can thus re-present both the going concerns of the practitioners and the local lexicon of accountability that all members have to learn in order to produce conducts which are observable-reportable (see Nicolini, 2009a for an in-depth discussion).

While the interview to the double in only one among many methods that could be used for this purpose, it is important to underscore that the zooming in does not try to access the values, beliefs, or presumed inner motives or which supposedly guide the conduct of the practitioners. The aim of the zooming in is, on the contrary, to surface the practical concerns which govern and affect all participants and a way to appreciate that from the perspective of

\footnotetext{
${ }^{8}$ In line with their Marxist legacy, CHAT authors tend to focus instead on the material reality and material implications of the object (see Engeström and Blackler, 2005, for a discussion).
} 
the members, practice unfolds in terms of an often pre-verbally experienced and yet collectively upheld sense of "what needs to be done".

To summarize, practical concerns are features of the practicing and not hidden mental contents or individual characteristics; as such, they constitute a further resource for re-

presenting practice. Zooming in here resembles less the magnification of an image and more the switching of theoretical lenses to allow for new aspects of the phenomenon to come to the fore. Combined with, and not an alternative to an ethnomethodologically-informed description, it helps us appreciate how the accomplishment of practice constitutes a recurrent and entrenched pattern of conduct moved by concerns that are deeply rooted in the moral character of members.

\section{Appreciating practice as bounded creativity}

The focus on the practical concerns that organise the accomplishment of practice also direct the attention towards the dynamic between poiesis and the bounded-ness of practice $-\mathrm{a}$ further focus of the zooming in. While practices need to be achieved each time for the first time (Garfinkel, 1967), so that practicing is fundamentally a poietic and creative affair, not "everything goes" in practice. Bounded-ness and poiesis coexist in uneasy tension.

The external boundaries of a practicing, so to speak, are in fact dependent upon what people agree needs to be done and what can be accounted for. These boundaries are expressed and sustained discursively through a local repertoire (the content) and lexicon (the right way of asserting it) of accountability. The repertoire and lexicon of accountability, like other features, are learned by novices through their socialisation. They establish discursively what is appropriate and what is not, what is admissible and what is not, what the correct style of practicing is and what is not. What is not understood, or what is not acceptable, does not belong to the practice and therefore needs to be dropped.

A further focus for the zooming in is thus the tension between repetition of the same and reproduction, what is indeterminate and what is bounded. Practice is indeterminate in that neither patients nor doctors behave in the same manner on any two occasions, and two telephone calls or meetings are never identical. Practicing is therefore inherently and necessarily an act of poiesis, creation, invention, and improvisation, aimed at producing sameness with what is, by definition, different and changeable: practices are literally reproduced on each novel occasion. At the same time, however, practicing is also bounded. 
The sense established by the practice is a horizon which prevents us from seeing things differently; analogously, the variety of ways in which we can creatively engage with the practical concerns set up by a practice are bounded by the limits imposed by the extant conditions and criteria of accountability.

The orientation, sense and accountability of a practice are, of course, specific to each historically-situated practice and are thus in continual evolution. This is because any assemblage of resources expresses and creates a set of practical concerns and, by making it possible, brings it about. However, even though these boundaries are continuously materially and discursively contested, debated and moved, they are always there.

Accordingly, a further focus for the zooming in is the tension between the drive to fulfil its perceived sense and the limits imposed by the extant conditions of accountability. We can focus both on how orientation, accountability and practical concerns conjure up the conditions of conduct by allowing practitioners to understand what is going on, what direction things ought to take, what is expected of them, and what is appropriate; or on the fact that these three aspects rarely coexist in harmony and tensions are common (Engeström, 1987; 2000). In both cases, the goal is to represent practices as dynamic, contested, and provisional affairs.

\section{Re-presenting practice by focusing on legitimacy and learning}

A further focus for the zooming in movement is provided by the consideration that a practice counts, as such, only for those who are capable of recognising it (Geertz, 1973; Giddens, 1984; De Certeau; 1984). "Recognising" is intended here both in a cognitive and normative sense. I may fail to recognise a practice either because I have no idea about what is going on (a sensation we have all experienced as novices in a certain practice) or because I do not want it or cannot accept it (because 'we do things differently here'). In both cases, however, as suggested originally by Wittgenstein (1953), the implication is that the notion of a private or arbitrary practice is a non-sense. Practices are by definition social, because it is only at this level that morality, meaning, and normativity can be sustained. For this reason, I have omitted the qualification "social" from the term "practice" in this paper. The term "social practice" says the same thing twice.

From the above it follows that all practices imply some level of durability and, in this sense, they carry traces, no matter how weak, of institutionalization. Practices differ from events in that they constitute enduring regimes of activity. As I noted before, this does not imply that 
practices are necessarily only replicated. In fact, the contrary applies and practice continuously changes, expands, and evolves. Therefore, the question for organisational scholars should not be "do practices change?" Instead, the question is the opposite - "through which mechanisms does practice achieve durability in time?"

It is my contention that to understand the durability and perpetuation of practices we should zoom in on at least four main aspects: learning, mediation, other people and other practices. Firstly, one can observe that methods for accomplishing practice, its orientation and normative force need to be learned. The sense and meaning of a practice are acquired in the social process through which novices become progressively proficient practitioners. It follows that if we are to provide a convincing account of both how ingrained ways of doing and saying persist in time and why people stick to them, we need to zoom in on learning. Without a coherent theory learning we are left with an obscure notion of practice as a hidden and metaphysical collective object that exerts some form of causal power over the behaviour of individuals ${ }^{9}$. As Jean Lave once put it ${ }^{10}$, without such an element, any account of practice is bound to collapse "like a table without a leg".

Secondly, practices are perpetuated and made durable by people who come to share similar skills, practical concerns, and ways of making themselves accountable. This concept could be expressed by the idea that all practices are sustained by a community (Lave and Wenger, 1991) as long as we agree that it is the practice which generates the social relations which emerge around it and not vice versa. Practice always generates sociality, but whether it also gives rise to recognisable and self-aware communities is dependent on local historical conditions. What is more important, however, is that the nature of the practical concerns and the boundary of the legitimate practices are continuously tested and con-tested within the social circle created by the joint endeavour. Questions of what is appropriate, what is legitimate, and what can be done are continuously tested in action so that practice is necessarily provisional and tied to specific historical and material conditions Thirdly, we can focus on the fact that the durability of practices derives from the tools and instruments that mediate it (see above). Tools and artefacts carry the script their designers embodied into them and for this reason they convey a particular culture of action. As a result, cultural artefacts constitute a means of transmission of social knowledge by carrying inscribed within them objectified norms of cognition, the assumptions on how work should

\footnotetext{
${ }^{9}$ See Turner (1984) for a discussion. Turner has the merit of raising this important issue, but then, by bundling all the practice theories together, he ends up 'throwing the baby out with the water'.

${ }^{10}$ Jean Lave made this remark during a presentation in Manchester in 1999.
} 
be carried out, and purposes of use. These all participate as formative elements in the practice itself (Miettinen, 1999). Tools mediate a historical and social dimension into the accomplishment of any practices. This renders every practice, even the most apparently solitary, a highly social and historical matter.

Finally, we can focus on the fact that the durability of practices is an effect of one practice becoming a resource in the accomplishment of other practices. Once it becomes part of a larger configuration, a practice is expected to be reproduced as a matter of course in order to contribute to the whole of which it is part. I will return to this idea shortly.

In summary, a final way to re-present and understand practice requires that we zoom in on the patterns of relationships among human individuals and how such patterns are learned and made durable. If, on one hand, this requires that we focus on the learned and mediated nature of practice, on the other, it points out that practice always occurs amid a texture of other interconnected practices that, while making possible the practice under scrutiny, also keep it in place. The attention is thus diverted from the practice itself to the texture of connection in which it is immersed. In turn, this type of attention requires that we widen our angle and zoom out to consider the field in which practices are carried out.

\section{ZOOMING OUT: TRAILING CONNECTIONS}

In the previous section I have suggested that practices are mainly unfolding orders of mediated conducts (sayings, doings and their various patterns) which constitute both the context and the result of such sayings and doings. Accordingly, re-presenting practice means observing and reporting the methodical ways in which practices are accomplished and the horizon of sense and landscape of artefacts amid which they unfold. This can be achieved through a palette of sensitising concepts and specific methods which make us see (and not see) particular aspects of the local accomplishment of a practice.

In this section I contend that examining in detail the local production of accountable order is only part of the job. Understanding and re-presenting practice also requires providing an appreciation of the connectedness of practice and the fact that activities never happen in isolation, so that practices are always immersed in a thick texture of interconnections. Telemedicine is a good case in point. Its accomplishment depends in fact on the work of a variety of people and on several other practices. These include not only the work of nurse and patients but also: the work and practice of the makers of the electronic device for transmitting 
the ECG; the work of the manufacturers of the computer used by the nurses; the work of the people in the lab who conduct the tests patients have to carry out before calling the centre, and so on. All practices thus depend on other practices. The zooming in shows, however, that the converse is also true. One practice often constitutes the resource for the accomplishment of others. Telemedicine contributes, for example, to the activity of the hospital where the nurses are based, the discipline of cardiology, and the Italian healthcare system.

In a sense, then, all practices are involved in a variety of relationships and associations that extend in both space and time and form a texture of dependencies and references.

Paraphrasing Latour (2005, p.44) we can state that practice is always a node, a knot and a conglomerate of many types of material and human agencies that have to be patiently untangled. For this reason, the study of practices cannot be limited to focusing on the details of their accomplishment. There is a need to integrate and alternate the zooming in movement with one which is horizon-widening and that, in accordance with the idea of zooming in, I would describe as zooming out on the texture of practice ${ }^{11}$.

Zooming out on practice requires thus moving between practice in the making and the texture of practices which causally connects this particular instance to many others. This can be achieved if we develop sufficient conceptual lenses and methodological devices to describe the ways in which practices are associated, form living assemblages, and produce effects and phenomena - from healthy patients to organised life, hierarchical and power inequalities and institutions. My suggested theoretical and methodological references for this task are the sociology of translation and ACT (Latour, 2005; Czarniawska, 2007). Authors working from this perspective have developed a sophisticated set of concepts and methodological recommendations to deal with the spatiotemporal "distributedness of ontological relations" and the consequent "dislocation of action" (Oppenheim, 2007, p. 477). These recommendations mostly centre around the injunction to follow the actors, tracing analytically the work that goes into making associations come about, and observing the effect produced by the ensuing socio-technical arrangement. The investigation can follow a compositional or decompositional strategy. Following the actors can thus literally mean shadowing human or non-humans actants and observing their daily activity of weaving connections (Czarniawska, 2007); or, alternatively, it may mean retracing the steps,

\footnotetext{
${ }^{11}$ CHAT authors evoke a similar idea when they suggest that practices cannot be understood in isolation and that the appropriate primary unit of analysis to understand human affairs is, in fact, "a historically-evolving collective activity system, seen in its network relations to other activity systems" (Engeström, 2000, p. 964).
} 
strategies and events that lead to the emergence, stabilisation or failure of specific assemblages using historical methods (Latour, 2005). When applied to the study of practices, this approach suggests trailing the associations between practices and identifying their effects. Taken together, these two approaches constitute an appropriate place to start the zooming out and an effective way to bring forward their inherent connectedness and ontological primacy in organisational affairs. In the next two paragraphs I briefly examine these two approaches in turn.

\section{Following the associations between practices}

Zooming out on practice to reveal and articulate its 'star shaped' (Latour, 2005, p.177) and inter-connected nature mainly requires to start with an empirical trailing of practices and their connections. This, in turn, requires two basic moves. Firstly, we need to uncover the connections between practices by following them in space and time. Secondly, we need to study how these connections are kept in place.

The first move (following the practice) can be accomplished using the methods and approaches of multi-site and focused ethnography (Marcus, 1995; Hannerz, 2003;

Knoblauch, 2005) or other techniques suitable to do field work on the move, such as shadowing, log studies and diaries (Czarniawska, 2007). Although these methods were originally developed to follow human and non-human actors, my experience is that they can be successfully extended to the study of practices. We can thus shadow the practice extending our observation to the different places where it shows up. The basic move here is to follow its intermediaries (people, artefacts and inscriptions) wherever they go. This would imply, for instance, following telemedicine patients through their clinical trajectories (Strauss and Glaser, 1975) from the moment when they are enrolled into the service until they are discharged; or following the tele-nurses through their professional apprenticeship. It would also require, for example, attending conferences, meetings, and gatherings where the practice is debated; attending policy-making forums where the practice is taken into consideration and sanctioned; reading the scientific and clinical literature where the practice is legitimated; travelling to other hospitals where the practice is adopted; and so on. This real-time shadowing of the practice could be integrated with a study of its emergence and evolution. As suggested by CHAT authors, an historical analysis allows us to build an appreciation of the unfolding material and political conditions that surrounded the birth of telemedicine and how these were inscripted in its current form (Engeström et al., 1999). As I have shown elsewhere, 
for example, the particular way of practicing telemonitoring used in my examples above emerged as the result of the incremental alignment of different interests over a period of several years, many of which left documentable traces (see Nicolini, 2009c for an extensive discussion).

By zooming out and combining real-time shadowing and historical reconstruction we can thus start building an appreciation of how local practices participate in larger configurations and how they enter as elements, ingredients, or resources in other activities.

The second move requires that we investigate how such associations are kept in place. The infra-language of the sociology of translation (Latour, 2005) is again very useful ${ }^{12}$. The concept of translation describes, in fact, "a relationship that does not transport causality but induces two mediators to co-exist" (Latour, 2005, p.108). The idea of translation invites us to appreciate that associations need to be "knotted" and kept actively in place through the coordination of humans and non-human mediators such as the therapy sheet, policy documents, plans, contracts and spaces. Only when all these resources are aligned to form chains of translation in such a way that the results of an activity are stabilised and turned into a more or less solid black box can effecting the activity of another practice be accomplished. This is exactly what tele-nurses discovered with dismay when their phones stopped working.

Uncovering the connections between practices by following them and studying how they are translated in space and time makes us see that the two-dimensional image of the trajectory (Strauss et al., 1982) is unfit for re-presenting and studying practice. To the extent that they extend rizhomatically in many directions, practices are better described in terms of practicenetwork; heterogeneous and "inter-textual" assemblages that participate with other, often larger, nets of action and which should always constitute the proper object of the study of practice.

\section{Zooming out by studying effects}

A second and related way of zooming out and extending the appreciation of practice beyond the here-and-now is to focus on the local and translocal effects produced by chains and assemblages of situated practices - what I called above practice-networks.

\footnotetext{
${ }^{12}$ According to Latour (2005) it constitutes an infra language in that it sets the scene for a performative material way of doing social science without however defining ex-ante the characters that will play on it.
} 
The example of telemedicine and the snapshots presented above are a good case in point. The star-shaped practice of caring for patients at a distance through skilled phone contacts performs, or more precisely contributes to, the performance of a variety of effects that go above and beyond deciding whether or not to allow patients to stay at home or to attend hospital. For instance, the practice under consideration engenders a specific way of being for both the interactants - what in social science jargon we could call two specific and peculiar 'identities'. In table 1 both the patients and the nurse start talking competently about a technical matter such as triglycerides, how to prevent 'artefacts' and setting medicine dosage - something one would not normally expect from either a frail patient or a cardiac nurse in Italy in the early 2000s, when nurses were not allowed to prescribe. The practice of telemedicine brings to bear an expert patient, an expert and intervening nurse, and a particular caring relation based on a mixture of self-control and control from a distance. Of course no identities, professional or non-professional, are supported by one event or activity such as the practice of making and responding to telephone calls. My argument, however, is that historical changes such as the emergence of the "expert patient" (Hardey, 1999) and its consequences for the way in which medicine is carried out can be understood in terms of the outcome of innumerable interconnected local practices like the one described here.

In a similar way, the new practice of telemedicine also modifies the existing pattern of empowerment in the field of healthcare. For example, the delegation of clinical responsibilities to nurses - a typical feature of telemedicine also observed by other researchers (Mort et al., 2003) can be seen as a form of erosion of the professional jurisdiction of doctors (or expansion of the nurses' jurisdiction). Analogously, over time there may be a tendency (as observed in my study) for the patient and the specialized centre to establish a direct and fiduciary relationship, so bypassing and sidelining other actors, including family doctors. This, in turn, might destabilise the existing entrenchment of existing practices, generating large and partially unknown effects, especially if and when the flow of funding will mirror the new flow of decision making. Again, although no single local practice can trigger such radical changes, it is possible (at least in principle) to describe and understand large scale changes in terms of a shift in the overall nexus of connected (healthcare) practices.

The examples help highlight why zooming out is fundamental for gaining an in-depth understanding of practice. From the vantage point of sitting near the nurse who takes care of patients one can only appreciate telemedicine as it happens and its immediate horizon of concern. One cannot "see" the many ways in which telemonitoring produces effects in the 
world. By changing conceptual lenses and moving in the field, however, we start to appreciate that the telephone calls and telemonitoring are implicated in the definition of what it means to be a patient or a nurse as well as, for example, the reshuffling of power in the hospital, health politics of the region where the telemedicine centre was based, and conceptions of a public health system. The appreciation provided by zooming out, however, also shows that the converse is true, and that the local practice of telemedicine is both enabled and constrained by event and practices taking place elsewhere. The controversy incited by the report of the tele-monitoring project when it was presented at one of the national cardiology meetings (during which doctors accused the centre of infringing legislation by giving nurses too much of a clinical role) and the political struggle to define which model of telemedicine would be reimbursed by the regional healthcare system both had significant and documentable consequences on the development of the local practice of telemedicine (see Nicolini, 2009c for an extensive discussion).

As before, in order to study empirically the connected-ness of practice through its effects we can employ the theoretical and methodological toolkit of the sociology of translation. The theory provides, in fact, an interesting explanation for the ways in which effects are translated in time and space. It does so by noting that mediators, which include boundary objects, names, protocols, plans, forms of categorisation and rules do not only support the association between practices. They also move it around acting as generalisers and localisers (Latour, 2005, p. 181). As generalisers, mediators summarise and black box the inherent multiplicity of practices, making them available as an object of work in another context; human experts "summarise" years of learning by doing, and artefacts such as the portable ECG black box the work and knowledgeability of all those who designed and built it. Through generalising mediators, large associations of practicing can become the objects of work of other, equally local and equally socio-material practices. It is through the mediatory work of these generalisers that local practices come to form what social scientists often describe as macro phenomena. Macro phenomena boil down to a complex texture of doings and sayings (meetings, conversations, debates, disputes), places (labs, offices), and objects (buildings, documents) which can all be observed proximally. Please note that this is also the crux of management. Managers are not omniscient and, of course, they cannot be everywhere at all times. They are finite people whose day job involves aligning black-boxed practices. From an articulative perspective, then, all social matters happen in a specific place and time, and macro or higher levels phenomena are just another way of referring to the locales and the effects of people trading in generalisers. Chairing the European Society of Cardiology or a 
large multi-national corporation does not require supernatural powers. Managers simply trade in generalisers.

Mediators can also act as localisers. In this role mediators act as a gateway through which large and stabilised practice-nets make themselves present in the local practicing. At line 21 and in table 1, the nurse and the patient refer to the procotol on how to measure accurately the urination. The protocol translates, in this local instance, the previous work, power and legitimacy of the vast practice-net of "scientific medicine". The notion of generaliser and localiser thus suggests that translation operates as two-way traffic. Just as the global can be explained as a nexus of locality, the local is itself fragmented and multiplied, a node in a complex nexus of actions that enter into it and that traverse it. Practice (including the practice of organising) is the result of this complex interplay between local and global.

In sum, by zooming out and trailing effects through their mediators (both generalisers and localisers) we can foreground and document how practices extend beyond the scope of the local set of practical concerns, so that locally accomplished practices become variably implicated in a variety of state of affairs which, in turn, may happen far from where the practicing takes place. Local practices such as tele-monitoring become thus a convenient starting point and a building block for explaining not only the local production of organised action and interaction, but also larger, more complex trans-local phenomena, such as the existence and functioning (the "organisation") of a ward, a hospital or a health authority, without contradicting the fundamental notion that practice is an oriented and concerned matter. By using empirical methods such as shadowing and historical analysis, and by employing notions such as that of translation and practice network, we can thus extend our articulative and practice-based investigation well beyond the accomplishment of practice and into the realm of the translocal and durable. Practice becomes in this way a convenient and usable ontological unit for making sense of a variety of organisational phenomena, from the existence of local forms of co-ordination to complex organisational arrangements such as large corporations, multinational firms, and other highly institutionalised forms such as markets (Knorr-Cetina, 2004). All without having to forfeit the idea that "it is practices all the way down" 13 .

\footnotetext{
${ }^{13}$ I am paraphrasing Geertz's story about an Englishman who, having been told by an Indian man that the world rested on a platform, which rested on the back of an elephant, which in turn rested on the back of a turtle, was asked what the turtle rested on. "Another turtle" was the response. "And that turtle?" rebuked the Englishman "Aha, Sahib", said the Indian, "after that it is turtles all the way down" (Geertz 1973: 28).
} 


\section{CONCLUDING REMARKS}

In this paper I have started to develop a lexicon and a method for interrogating practices as constitutive of organisational and social phenomena. Using suggestions derived from different social science traditions I argued that the task can be captured by the image of "zooming in on" and "zooming out of" practice obtained through magnifying or blowing up the details of practice, switching theoretical lenses, and selective re-positioning so that certain aspects are fore-grounded and others are temporarily sent to the background. The effort should always start with a focus on practice-making and the concerted accomplishment of a real-time activity. This, however, should be followed by, and alternated with, an attempt to trail the active ways in which practices are associated. The aim is to get an appreciation of the wider texture made up of their mutual implications and to grasp the mutual relationships between the local real-time accomplishment of practices and the texture that they form and in which they are implicated. By recursively navigating between local instances and their connections, we can start gaining an appreciation that large organisational phenomena are but one of the effects of this seamless web of ongoing practice. The framework, which is necessarily provisional and open-ended, is summarised in table 3.

Insert table 3 about here

The framework is based on a number of sensitising concepts which constitute a constructive vocabulary that generate practices (and practicing) as open-ended, recognizable sets of mediated doings and sayings which unfold and are accomplished in view of the fulfilment of a horizon of sense and a set of normative practical concerns. These continuously-evolving organised sets of doings and sayings are obtained through use of a variety of resources and they produce a variety of effects. Because, through a process of translation, the effects produced in one practice are used as resources or signs for others, practices constitute a vast, intricate texture of mutual imbrications and deferrals: a practice-net. Moreover, practices and their mutual relationships always instantiate a particular configuration of interests and involve phenomena such as hierarchy, power, and identity. As activity is in some party's interest, practices need therefore to be established and stabilised through a process of translation. This is always, by definition, a political endeavour. 
The framework I proposed brings together and enriches aspects derived from various historical ways of understanding practice. It builds on the many strengths of the existing practice theories using them together, instead of playing them against each other. My approach is thus intentionally eclectic for, in my view, the complexity of practice cannot be ever captured by a single totalising discourse and is, instead, better addressed through a toolkit logic.

While it constitutes a promising start, the framework proposed here is still in an embryonic state and is in need of development in several directions. In the first place, more work will be necessary to document in detail how practices hang together. While the vocabulary of the sociology of translation is a useful point of departure, further empirical work is necessary to shed light on the alignment and co-ordination of practices, how they form assemblages, and how these assemblages are made durable and change overtime.

Secondly, more work will be needed to document how stabilised assemblages of practices affect the local activity. Although there is no need to rediscover the idea of retro-action (which, say Hacking (2004), has been sufficiently re-discovered) there is a need to examine better how arrangements of practices make themselves present in the daily routine of practitioners, so that we can authentically appreciate what I called the two-way street relation between local practices and their assemblages.

Thirdly, and closely related, the framework invites us to carry out further work on the relationships between practice and institution (and between practice-based and institutional theories). The framework developed above explicitly suggests that the institutional texture of social and organisational life is as an artefact and a provisional effect that is both the condition and the outcome of the practicing. How this happens will need to be explored in further detail. Interestingly, this concern has also been raised by neo-institutional authors such Lounsbury and Ventresca (2003), Lawrence and Suddaby (2006), and Lounsbury and Crumley (2007) so that practice promises to constitute a fertile ground for future conversation between existing approaches within organisation studies. 


\section{REFERENCES}

\section{Bakhtin, Mikhail M.}

1981 The dialogic imagination: Four essays. (Transl. C. Emerson \& M. Holquist). Austin: University of Texas Press.

Barley, Stephen, and Gideon Kunda

2001 'Bringing Work Back In’ Organization Science 12(1): 76-95.

Belova, Olga, Ian King, and Martyna Sliwa

2008 'Introduction: Polyphony and Organization Studies: Mikhail Bakhtin and Beyond' Organization Studies 29(4): 493-500.

Blackler, Frank

1993 'Knowledge and the theory of organizations: organizations as activity systems and the reframing of management' Journal of Management Studies 30(6): 863-84.

Boden, Deidre

1994 The Business of Talk: Organizations in Action. Cambridge: Polity Press.

Bourdieu, Pierre

1990 The Logic of Practice. Cambridge: Polity Press. (or. ed. 1980).

Certeau, Michael de

1984 The Practice of Everyday Life. Berkeley: University of California Press.

Chia, Robert, and Robin Holt

2006 'Strategy as Practical Coping: a Heideggerian Perspective' Organization Studies 27(5): 635-655.

Crabtree, Andrew

2001 'Doing Workplace Studies: Praxiological Accounts-Lebenswelt Pairs', TeamEthno Online, Mike Rouncefield (ed.) Issue 1 (ISSN 1475-0872).

Czarniawska, Barbara

2004 'On time, space, and action nets' Organization 11(6): 773-791. 
Czarniawska, Barbara

2007 Shadowing: And Other Techniques for Doing Fieldwork in Modern Societies.

Copenhagen: Liber and Copenhagen Business School Press.

Czarniawska, Barbara, and Tor Hernes editors

2005 Actor-Network Theory and Organizing. Copenhagen: Liber and Copenhagen

Business School Press.

Engeström, Yrjö

1987 Learning by expanding: An activity- theoretical approach to developmental research. Helsinki: Orienta-Konsultit.

Engeström, Yrjö

2000 'Activity theory as a framework for analysing and redesigning work' Ergonomics 43 (7): 960-974.

Engeström, Yrjö, and Frank Blackler

2005 'On the life of the object' Organization 12(3): 307-330.

Engeström, Yrjö, Rejio Miettinen, and Raija-Leena Punamäki editors

1999 Perspectives on Activity Theory. Cambridge: University Press.

Garfinkel, Harold

1967 Studies in Ethnomethodology. Englewood Cliff: Prentice Hall.

Garfinkel, Harold, and Harvey Sacks

1970 'On formal structures of practical action'. In Theoretical sociology: perspectives and developments. J. C. McKinney and E. A. Tiryakian (eds): 338-66. New York: AppletonCentury-Crofts.

Geertz, Clifford

1973 The interpretation of cultures. New York: Basic Books.

Gherardi, Silvia

1995 'When Will He Say: 'Today the Plates Are Soft'? The Management of Ambiguity and Situated Decision-Making' Studies in Cultures, Organizations and Societies 1(1): 927.

Giddens, Anthony

1984 The Constitution of Society. Cambridge: Polity Press. 
Gubrium, Jaber F.

1988 Analyzing Field Reality. Thousand Oaks: Sage.

Hacking, Ian

2004 'Between Michel Foucault and Erving Goffman: between discourse in the abstract and face-to-face interaction' Economy and Society 33(3): 277-302.

Hannerz, Ulf

2003 'Being There... And There... And There! Reflections on Multi-Site Ethnography'

Ethnography 4(2): 201-16.

Hardey, Michael

1999 'Doctor in the house: the Internet as a source of lay health knowledge and the challenge to expertise' Sociology of Health \& Illness 21(6): 820-835.

Hardy, Cynthia

2004 'Scaling up and Bearing Down in Discourse Analysis: Questions Regarding Textual Agencies and Their Context' Organization 11(3): 415-425.

Heidegger, Martin

1947/1993 'Letter on Humanism'. In Heidegger. Basic Writings. David F. Krell. Ed.: 213-

39. New York: Harper \& Row.

Heritage, John

2004 'Conversation analysis and institutional talk: analyzing data'. In Qualitative

Research: Theory, Method and Practice. 2nd Edition. David Silverman (ed.): 161-182.

London: Sage.

Hilbert, Richard

1990 'Ethnomethodology and the Micro-Macro Order', American Sociological Review 55:

794-808.

Iedema, Rick

2007 'On the Multi-modality, Materially and Contingency of Organization Discourse' Organization Studies 28(6): 931-946.

Kaptelinin, Victor, and Bonnie A. Nardi

2006 Acting with technology: Activity theory and interaction design. Cambridge, MA:

MIT Press. 
Knoblauch, Huber

2005 'Focused Ethnography’ Forum Qualitative Sozialforschung / Forum: Qualitative Social Research, North America, 6, sep. 2005. Available at: http://www.qualitativeresearch.net/index.php/fqs/article/view/20. Date accessed: 02 May. 2009.

Knorr Cetina, Karin, and Alex Preda

2004 The Sociology Of Financial Markets. Oxford: University Press

Latour, Bruno

2005 Reassembling the social. Oxford: OUP.

Lave, Jean, Etienne Wenger

1991. Situated Learning: Legitimate Peripheral Participation. New York: Cambridge University Press.

Lawrence, Thomas B., and Roy Suddaby

2006 'Institutions and institutional work' in Sage Handbook Of Organization Studies, 2nd ed. Stewart Clegg, Cynthia Hardy, Walter R. Nord, and Tom Lawrence (eds) : 215-254.

Thousand Oaks: Sage.

Levinson, Stephen C.

2005 'Living with Manny's dangerous idea' Discourse Studies 7: 431-453.

Lindberg, Kajsa, and Barbara Czarniawska

2006 'Knotting the action net, or organizing between organizations' Scandinavian Journal of Management 22(4): 292-306.

Llewellyn, Nick

2008 'Organization in Actual Episodes of Work: Harvey Sacks and Organization Studies' Organization Studies 29(5): 763-791.

Llewellyn, Nick, and Jon Hindmarsh, editors

2009 Organization, interaction and practice: Studies of real time work and organizing.

Cambridge: Cambridge University Press.

Lounsbury, Michael, and Ellen T. Crumley

2007 'New Practice Creation: An Institutional Approach to Innovation' Organization Studies, 28(7): 993-1012.

Lounsbury, Michael, and Marc J. Ventresca

2003 'The new structuralism in organizational theory' Organization 10: 457-480. 
Luff, Paul, Jon Hindmarsh, and Christian Heath

2000 Workplace studies: Recovering work practice and informing system design.

Cambridge: Cambridge University Press

Lynch, Michael

2001 'Ethnomethodology and the logic of practice'. In The practice Turn in Contemporary

Theory. T. Schatzki, K. Knorr Cetina and E. Von Savigny (eds) London: Routledge.

Lynch, Michael

1993 Scientific Practice and Ordinary Action: Cambridge: University Press.

MacIntyre, Alasdair

1981 After Virtue. A Study in Moral Theory. London: Duckworth.

Marcus, George

1995 'Ethnography in/of the World System: The Emergence of Multi-Sited Ethnography' Annual Review of Anthropology 24: 95-117.

McHoul, Alex

1986 'The getting of sexuality: Foucault, Garfinkel and the analysis of sexual discourse'

Theory, Culture and Society 3: 65-79.

Mehan, Hugh

1985 'The Structure of Classroom Discourse'. In Handbook of Discourse Analysis, Vol. 3. Teun A. Dijk (ed.) : 120-131. New York: Academic Press.

Miettinen, Reijo

2005 'Object of activity and individual motivation' Mind, Culture, and Activity 12(1): 5368.

Miettinen, Reijo

1999 'The riddle of things. Activity theory and Actor Network Theory as approaches of studying innovations' Mind, Culture, and Activity 6 (3): 170-195.

Miettinen, Reijo, Jaakko Virkkunen

2005 'Epistemic objects, artefacts, and organizational change', Organization 12(3): 437456.

Mort, Maggie, Carl May, and Tracy Williams

2003 'Remote Doctors and Absent Patients: acting at a distance in telemedicine' Science, Technology \& Human Values 28(2): 274-295. 
Nicolini, Davide

2006 'The work for making Telemedicine work' Social Science \& Medicine, 62: 27542767.

Nicolini, Davide

2007 'Stretching out and expanding medical practices. The case of telemedicine' Human Relations 60(6): 889-920.

Nicolini, Davide

2009a 'Articulating Practice through the Interview to the Double' Management Learning, 40 (2): 195-212.

Nicolini, Davide

2009b 'A Theory-Method Package for Turning the Ethnographic Gaze on Work and Organizational Practices'. In Organizational Ethnography. Studying the complexity of everyday life. S. Ybema, D. Yanow, H. Wels, F. Kamsteeg (eds.): 120-138. London:

SAGE.

Nicolini, Davide

2009c 'Medical innovation as a process of translation. A case from the field of telemedicine' British Journal of Management, in press.

Oppenheim, Robert

2007 'Actor-network theory and anthropology after science, technology, and society' Anthropological Theory 7: 471.

Orr, Julian

1996 Talking about Machines: An Ethnography of a Modern Job. Ithaca: Cornell University Press.

Ortner, Sherry.

1984 'Theory in anthropology since the 60s' Comparative Studies in Society and History 26(1): 126-166.

Rawls, Anne W. 2008 'Harold Garfinkel, Ethnomethodology and Workplace Studies' Organization Studies 29: 701-732. 
Reckwitz, Andreas

2002 'Toward a Theory of Social Practices. A Development in Culturalist Theorizing' European Journal of Social Theory 5(2): 243-263.

Samra-Fredericks, Dalvir

2003 'Strategizing as Lived Experience and Strategists' Everyday Efforts to Shape Strategic Direction' Journal of Management Studies 40(1): 141-174.

Samra-Fredericks, Dalvir 2004 'Understanding the production of 'strategy' and 'organization' through talk amongst managerial elites' Culture \& Organization 10(2): 125-141.

Samra-Fredericks, Dalvir 2005 'Strategic Practice, 'Discourse' and the Everyday Interactional Constitution of 'Power Effects' Organization 12: 803-841.

Samra-Fredericks, Dalvir, Reijo Miettinen, and Dvora Yanow 2005 'Re-turn to Practice: Understanding Organization As It Happens' Call for paper for the $2^{\text {nd }}$ The Second Organization Studies Summer Workshop.

Schatzki, Theodor, Karen Knorr Cetina and Eike Von Savigny editors 2001 The Practice Turn in Contemporary Theory. London: Routledge

Schatzki, Theodore 2001 'Practice Mind-ed Orders'. In The practice Turn in Contemporary Theory. T. Schatzki, K. Knorr Cetina and E. Von Savigny (eds). London: Routledge.

Schatzki, Theodore 2002 The site of the social: A philosophical exploration of the constitution of social life and change. University Park, PA: The Pennsylvania State University Press.

Schatzki, Theodore 2005 'The Sites of Organizations' Organization Studies 26: 465-484.

Schatzki, Theodore

1996 Social practices: A Wittgenstenian approach to Human activity and the Social. New York: Cambridge University Press.

Shotter, John

1993 Conversational Realities: Constructing Life through Language. London: Sage 
Silverman, David

1985 Qualitative Methodology \& Sociology. Aldershot: Gower.

Strauss, Anselm, and Barney Glaser

1975 Chronic Illness and the Quality of Life. St Louis, MO: Mosby.

Strauss, Anselm, Shizuko Fagerhaugh, Barbara Suczek, and Carolyn Wiener

1982 'Sentimental work in the technologized hospital' Sociology of Health and Illness 4(3): 254-77.

Streeck Jurgen, and Siri Mehus

2004 'Microethnography: the Study of Practices'. In Handbook of Language and Social Interaction. K. L. Fitch and R. Sanders (ed.): 381-404. Mahwah, NJ: Lawrence Erlbaum Associates.

ten Have, Paul

1999 Doing Conversation Analysis. London: Sage.

Thévenot, Laurent

2001 'Pragmatic Regimes Governing the Engagement with the World'. In The Practice Turn in Contemporary Theory. T. Schatzki, K. Knorr Cetina and E. Von Savigny (eds). London: Routledge.

Turner, Sthephen

1994 The Social Theory of Practices: Tradition, Tacit Knowledge, and Presuppositions. Cambridge: Polity Press.

Wittgenstein, Ludwig

1953 Philosophical Investigations. Oxford: Blackwell

Wittgenstein, Ludwig

1980 Culture and Value. Collected and edited by G. Von Wright, translated by P. Winch. Oxford: Blackwell.

Yanow, Dvora

2006 'Talking about Practices: On Julian Orr's Talking About Machines' Organization Studies 27(12): 1743-1756.

Ybema, Sierk, Dvora Yanow, Harry Wels, and Frans Kamsteeg 2009 Organizational Ethnography. Studying the complexity of everyday life. London: Sage. 


\section{Appendix 1}

List of the standard Jefferson notation system symbols used in table 2 (please note this is not a complete list of symbols). For a discussion of the notation system see ten Have, P (1999).

$\begin{array}{ll}\begin{array}{l}\text { (.) Just noticeable pause } \\ \text { wor - } \\ \text { wo::rd }\end{array} & \text { A dash shows a sharp cut-off } \\ \text { word }= & \text { The equals sign shows that there is no discernible pause between two } \\ =\text { word } & \text { speakers' turns } \\ \text { word } & \text { Underlined sounds are louder }\end{array}$
A: word [word Square brackets aligned across adjacent lines denote the start of B: $\quad$ [word overlapping talk.


$1 \mathrm{~N}$ : Good morning, how are you?

2 P: Not too bad, thank God....however, you

3 know....my triglycerides ${ }^{14}$

4 N: I know....I've seen your tests....

5 P: Yeah....my triglycerides!

6 N: They are not exactly where they should be....

7 P: (Mumble)....well, you know, I cannot resist....

8 N: I know....some crisps here....some cheese

9 there....and your triglycerides stay high.

10 Can you tell me your pressure, please?

11 P: 90 over 130

$12 \mathrm{~N}$ : Seems fine to me...Did you measure it in the

13 morning?

14 P: Yes, yes

$15 \mathrm{~N}$ : How about your weight?

16 P: Well...uh....eighty....eighty two kilos....

17 N: Eighty or eighty-two kilos?

18 P: Eighty-two (in a low voice) but it varies ${ }^{15} \ldots$...

$19 \mathrm{~N}$ : You'll have to check your diuresis....two
The nurse takes a look at the sheet

with the tests in front of her/him

which he/she had looked at

before. Then he/she puts down the

sheet and smiles (on the phone)...
While making this comment, the nurse pulls up the therapy sheet and takes a look at the data (the sheet also contains data from previous calls)

The nurse turns slightly on the chair and smiles... she looks at the sheet and writes down the information.

The nurse looks at the folder

\footnotetext{
${ }^{14}$ Level of fat in the blood

${ }^{15}$ Patients with serious heart failure can retain water at an outstanding rate. For this reason diuretics constitutes one of the main medications for this condition and control of urination is crucial.
} 
kilos are

20 quite a lot....remember how to do it, right?

21 When you go to the toilet you...

22 P: (interrupting the nurse) Yeah, I remember....I

$23 \quad$ KNOW it well

$24 \mathrm{~N}$ : But you'll have to be more strict with your

25 diet....no crisps....too much salt, and no

26 cheese either, or at the most a little bit.

[The rest of the call is omitted for reasons of space]

Table 1: Partial transcription of a call between the telemedicine centre and a patient 
1 Good morning how are you?

2 Not too bad thank God (.)

3 however (.) you know

3 (.) my triglycerides ${ }^{16}=$

$4 \quad=$ I know (.) I've seen your tests

5 (.) Yeah::my triglycerides $=$

$6 \quad=$ They are not exactly where they should be (.)

$7 \mathrm{~mm}($.) well you ::know:: I cannot resist=

$8 \quad=I$ know (.) some crisps here some cheese there::

9 and your triglycerides stay high (.)

10 Can you tell me your pressure, please?

1190 over 130

12 Seems fine to me ::

13 Did you measure it in the mor[ning?

14 [Yes:: yes

15 How about your weight?

16 Well::uh (.) eighty uh (.) eighty two kilos::

17 Eighty or eighty-two kilos?

Table 2: zooming in on the call in table 1

${ }^{16}$ Level of fat in the blood 


\section{Zooming in}

Focus on / articulate the: $\left\{\begin{array}{l}\text { Sayings and doings } \\ \text { Active role of material elements and infrastructure } \\ \text { Local methods and micro strategies of concerted } \\ \text { accomplishment } \\ \text { Body choreography } \\ \text { Practical concerns } \\ \text { Sense and object of the practice } \\ \text { Local repertoire and lexicon of accountability } \\ \text { Conditions of legitimacy } \\ \text { Sociality and socialisation process } \\ {[\ldots]}\end{array}\right.$

\section{Zooming out}

Follow the practice and articulate the:
Associations between practices and the resulting practicenet

Reciprocal implications (how one practice becomes the resource for other ones)

Mediators

Patterns of associations and interests (practice -net)

Local and trans-local (global) effects

Effects of the global on the local

$[\ldots]$

Table 3: Summary of the proposed framework 